\title{
A genome-wide analysis reveals that the Drosophila transcription factor, Lola, promotes axon growth in part by suppressing expression of the actin nucleation factor, Spire
}

Michael A Gates ${ }^{1,2,3,4+}$, Ramakrishnan Kannan ${ }^{1,2+}$ and Edward Giniger ${ }^{1,2,3,4^{*}}$

\begin{abstract}
Background: The phylogenetically conserved transcription factor Lola is essential for many aspects of axon growth and guidance, synapse formation and neural circuit development in Drosophila. To date it has been difficult, however, to obtain an overall view of Lola functions and mechanisms.

Results: We use expression microarrays to identify the lola-dependent transcriptome in the Drosophila embryo. We find that lola regulates the expression of a large selection of genes that are known to affect each of several loladependent developmental processes. Among other loci, we find lola to be a negative regulator of spire, an actin nucleation factor that has been studied for its essential role in oogenesis. We show that spire is expressed in the nervous system and is required for a known lola-dependent axon guidance decision, growth of ISNb motor axons. We further show that reducing spire gene dosage suppresses this aspect of the lola phenotype, verifying that derepression of spire is an important contributor to the axon stalling phenotype of embryonic motor axons in lola mutants.

Conclusions: These data shed new light on the molecular mechanisms of many lola-dependent processes, and also identify several developmental processes not previously linked to lola that are apt to be regulated by this transcription factor. These data further demonstrate that excessive expression of the actin nucleation factor Spire is as deleterious for axon growth in vivo as is the loss of Spire, thus highlighting the need for a balance in the elementary steps of actin dynamics to achieve effective neuronal morphogenesis.
\end{abstract}

\section{Background}

Transcriptional regulation is central to the development of neural connectivity [1-4]. First, by controlling the identities and differentiation of individual neurons, and of the non-neuronal cells that often provide them with growth and guidance cues, it specifies patterns of axon growth and dendritic ramification. For example, classic homeotic selector genes produce segment-specific patterns of axon projection by controlling transcription of key cell fate and guidance molecules [5,6]. At a higher level of specificity, the axonal projections of many

\footnotetext{
* Correspondence: ginigere@ninds.nih.gov

+ Contributed equally

'Basic Neuroscience Program, National Institutes of Neurological Disorders and Stroke, National Institutes of Health, Bethesda, MD 20892, USA

Full list of author information is available at the end of the article
}

individual neurons and classes of neurons are determined by the expression of particular combinations of transcription factors. Perhaps the best-characterized example of this is the 'code' of LIM domain transcription factors that controls the dorsal versus ventral projection of motoneurons from the central nervous system (CNS) in both vertebrates and invertebrates [7-9]. Thus, in Drosophila, for instance, the Lim/Islet/Eve-dependent pattern of motor axon projection arises in part from regulated transcription of a critical axon guidance receptor, the Netrin receptor Unc5 [10]. In addition to specifying axon projection by defining cell identity per se, transcriptional regulation is also key to determining more local properties of axon patterning. For example, recent experiments have shown that the attractive midline receptor DCC in addition to controlling cytoplasmic

\section{C) Biomed Central}


signal transduction and growth cone motility directly, further contributes to midline axon guidance by inducing expression of commissureless mRNA [11].

Beyond simply expressing the right proteins, however, a neuron must express these proteins at the proper relative levels such that it responds appropriately to precise ratios of specific external and internal cues [12-16]. Again, tight regulation of gene expression is essential to make this happen. The Drosophila transcription factor gene lola is an exemplar of this latter role of transcriptional regulation. Thus, for example, lola orchestrates midline crossing by CNS axons by coordinately regulating the expression of both the midline repellant Slit and also its axonal receptor, Robo [17]. The effects of lola on slit and robo expression individually are modest, but in combination, the cumulative effect is profound, and further enhanced by lola-dependent regulation of other components of the pathway (robo2 and robo3; HJ Song, BJ Taylor and EG, unpublished observations). Similarly, detailed analysis of mutant phenotypes suggested that lola is likely to be a 'master regulator' of the patterning of the ISNb motonerve of Drosophila, coordinately regulating expression of the suite of guidance proteins responsible for ISNb motoneuron/muscle interaction [18].

In addition to its well-characterized functions during early embryonic axonogenesis, lola is also involved in construction or function of neural circuits throughout the lifespan of the fly. This includes dendritic morphogenesis in embryonic sensory neurons [19] and both dendritic and axonal development of adult olfactory projection neurons [20]. Moreover, lola has been identified in a number of genetic and genomic screens for genes involved in complex behaviors, including locomotion, alcohol sensitivity, mating and aggression [21-24]. While the role of lola in these processes is less well understood than is its well-studied function in axon patterning, the varied effects of lola underscore its importance for establishing patterns of gene regulation that produce a properly functioning nervous system. Beyond its neuronal functions, lola also plays key roles in several nonneuronal tissues. For example, lola is essential for oogenesis, in part due to its regulation of programmed cell death at different stages of oogenesis [25].

The lola locus encodes a family of more than 20 transcription factor isoforms by alternative splicing [26-28]. All share a BTB dimerization domain at the amino terminus, allowing co-expressed Lola proteins in a cell to form a variety of heterodimeric species in vivo. At least 17 lola isoforms encode $\mathrm{Zn}$ fingers in their variant 3' exons. While some isoforms have a single $\mathrm{Zn}$ finger, in most isoforms, the $\mathrm{Zn}$ fingers are present as a pair, with an amino-terminal $\mathrm{CCHC} \mathrm{Zn}$ finger that is apt to be a protein interaction domain, followed by a $\mathrm{C} 2 \mathrm{H} 2 \mathrm{Zn}$ finger that is likely to bind nucleic acid [26]. Biochemical experiments reveal that isoform $\mathrm{K}$ binds directly to the transcriptional enhancer of the copia retrotransposon, activating its expression in transient transfection assays [29]. A different isoform, isoform $\mathrm{T}$, has a nearly identical sequence as isoform $\mathrm{K}$ in its $\mathrm{Zn}$ fingers and binds the same DNA sequence, but it suppresses copia expression, competing with isoform $\mathrm{K}$ in co-transfection experiments. Consistent with these evidently antagonistic effects of lola isoforms, expression of the endogenous copia is upregulated in the CNS of lola mutants, but downregulated in the gonads of the same individuals.

To better understand the regulation of axon growth and guidance by lola, we performed a microarray study to identify genes whose transcript level was altered in a lola mutant. In addition to the sole known direct target of Lola, the retrotransposon copia, we found changes in expression level of a substantial number of genes that have been implicated previously in well characterized lola-dependent developmental events, including axon patterning, eye development, Notch signaling and programmed cell death. Among the affected genes was an unexpected downstream target, the gene encoding the actin nucleation factor Spire, which was upregulated in the lola mutant. spire is required for both anteroposterior and dorsoventral patterning of the developing oocyte [30], but its zygotic functions have never been characterized. We found that mutation of spire by itself caused defects in a lola-dependent axon patterning event, extension of the ISNb motonerve, and further that reduction of spire expression, by introduction of a heterozygous spire mutation, suppressed the axonal defects of lola in extension of the ISNb motonerve. These data suggest that overexpression of spire makes a significant contribution to the ISNb axonal phenotype of lola.

\section{Results}

\section{Design of the microarray experiment}

We set out to use cDNA microarray analysis to profile the changes in gene expression in lola mutant embryos relative to wild type. For detailed protocol see Materials and methods and [31], but key points are as follows. Since our primary interest was in axon patterning, we collected embryos at 10 to 12 hours after egg laying, a time when many axons are extending along lola-dependent nerve pathways in the embryo. We employed a molecular-null lola allele, lola ${ }^{\text {ORE76, which introduces a }}$ stop codon early in the common region of lola and does not accumulate detectable levels of any Lola protein fragment in vivo. Seven pairs of mutant and control wild-type RNA samples were collected independently, with each sample derived from approximately 300 embryos, and each pair collected in parallel on the same day. Homozygous mutant embryos were positively selected in the fluorescent microscope using GAL4- 
driven expression of a green fluorescent protein $(G F P)$ marker in the mutants, and controls were selected in a parallel cross employing the same GAL4 and GFP markers. Labeled first-strand cDNA programmed from experimental and control samples were co-hybridized to spotted cDNA arrays bearing the Drosophila Gene Collection (release 1 and release 2), prepared for the Northwest Drosophila Microarray Consortium (Gene Expression Omnibus Platform GPL 1908) [32,33]. For four pairs of samples, the experimental probe was labeled with $\mathrm{Cy}-3$ and control with $\mathrm{Cy}-5$, while dye assignments were reversed for the remaining three sample pairs.

Drosophila arrays had 12, 144 features (that is, cDNA spots), of which 10, 376 were analyzed statistically for differential expression. Data processing and statistical analysis were performed using the limma statistical package $[34,35]$. Standard errors were modified using an empirical Bayes method as implemented in limma. Adjustment for multiple testing was performed by controlling the false discovery rate to 5\% [36]. Selection of features as showing differential expression was based solely on statistical significance: no $a$ priori assumptions were made as to minimum expression level or minimum fold-change in expression. This analysis identified 597 genes that showed differential expression between mutant and control (displayed graphically in Figure 1; complete list in Table S1 in Additional file 1), with significant fold changes as low as 1.14-fold.

\section{Expression of copia and other retrotransposons is altered in lola mutants}

Inspection of the features that passed our analysis revealed that six among those with the largest fold change ( 4.2 to 4.6 ) corresponded to six independent cDNAs of the retrotransposon copia. copia is the only verified direct target of Lola, and bears Lola binding sites in its enhancer. lola isoform K activates copia expression in cultured cells, and in situ hybridization reveals that copia RNA levels are altered in lola mutant embryos [29]. Quantitatively, all six features corresponding to copia showed a similar factor of increase in expression level in the mutants, and these six cDNAs were the only features on the array that corresponded to copia. In addition to copia, we also detected significant expression changes in features corresponding to several

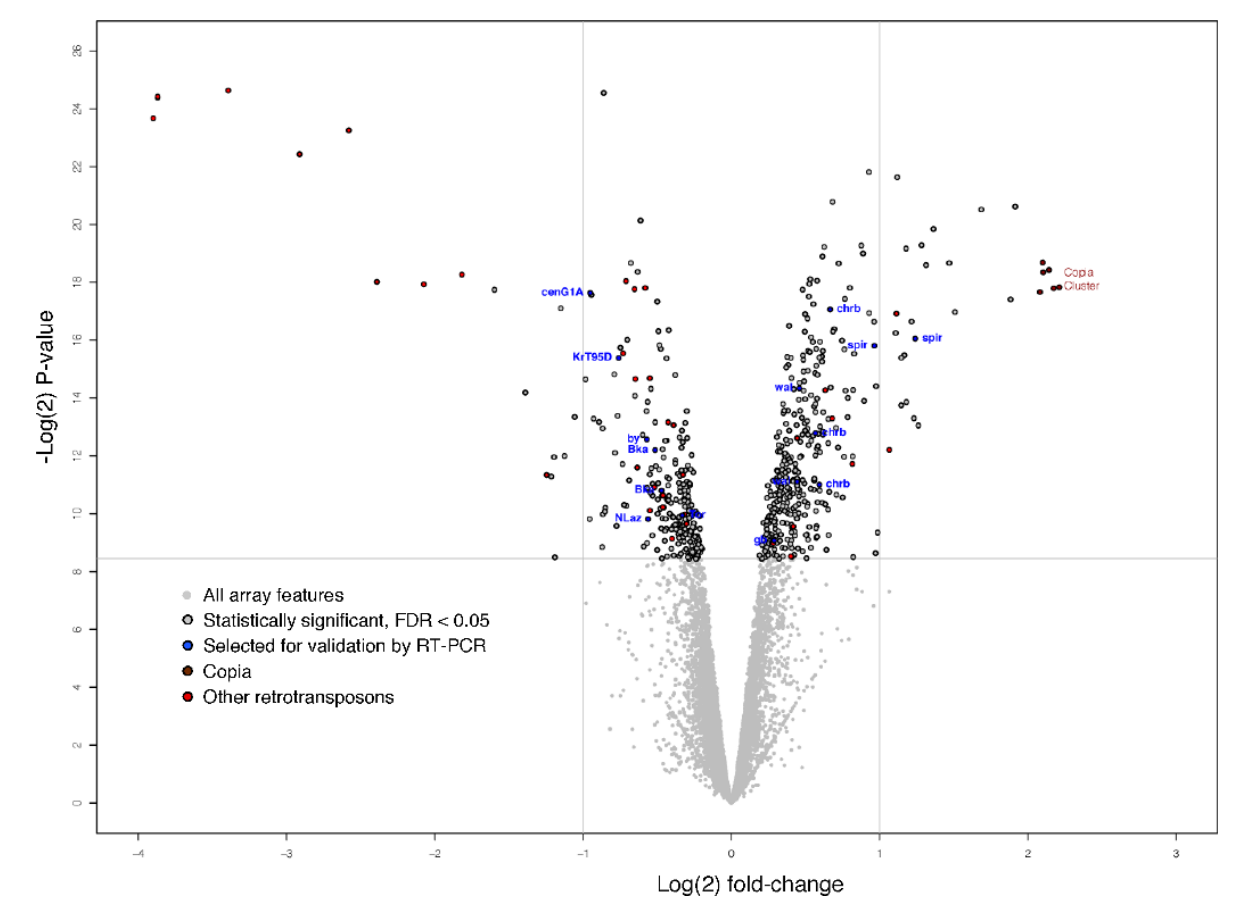

Figure 1 Distribution of effects on transcript accumulation in lola microarrays. Graphical representation of data from lola microarray experiment. Each gray dot represents the mean ratio of normalized feature hybridization for lola mutant/wild type from seven experiments, presented as a scatter plot of log2(adjusted $P$-value) versus log2(fold change) for each cDNA. Points above the horizontal line (circled in black) are statistically significant with the false discovery rate controlled to 0.05 (which, for this analysis, roughly corresponded to adjusted $P<0.0028$ ). The six cDNAs corresponding to the copia retrotransposon are highlighted in brown; other retrotransposon transcripts are in red. Features corresponding to loci also quantified by quantitative RT-PCR are in blue. Note that different cDNAs corresponding to a single locus are plotted separately; thus, for example, there is a cluster of six, independent brown spots for copia. 
other retrotransposons, including stalker, stalker2, 297 and roo. Whether these are also direct Lola targets or indirectly dependent on lola remains to be examined.

\section{Validation of positives from the array analysis}

Two lines of evidence support the validity of the positives from the array analysis. First, we performed quantitative RT-PCR (qRT-PCR) of ten genes with statistically significant changes in expression level between mutant and control. To give the best assessment of the overall reliability of the dataset, we selected genes that showed a range of fold change (1.2 to 2.4), and we excluded retrotransposons, which showed the largest fold changes in the dataset (Figure 1). Of the ten genes selected, five were validated in the qRT-PCR as changed in level in RNA samples from the lola null mutant $(P<0.05$; Table $1)$. We suggest that this provides a minimum estimate of the reliability of the microarray dataset for two reasons. First, the cDNA representing a particular gene on the array and the associated qRT-PCR target in the validation experiment may not query the same splice variant or set of splice variants, and thus may be regulated differently. Second, the RNA for the array analysis was derived, in aggregate, from 2, 100 embryos per genotype while the qRT-PCR samples were derived from 150 to 250 embryos. The substantially larger size of the sample contributing to the array analysis, therefore, may have materially reduced its variance compared with the qRT-PCR.

As a second validation, the results of the array analysis were also supported by an independent microarray experiment. Expression profiling was performed for a different kind of lola mutation, the allele lola ${ }^{\mathrm{ORC} 4}$ that inactivates only a single lola isoform, lola $K[26,31]$.
When we examined the expression profile of lola ${ }^{O R C 4}$ mutant embryos versus their matched control samples, and limited our statistical analysis to the set of 597 features with significantly altered expression in the lola null mutant, we found that 204 of these features also showed differential expression in the lola ${ }^{\text {ORC4 }}$ dataset (false discovery rate controlled at 0.01; Figure 2). In contrast, in 500 simulations in which 597 features were selected at random from the lola ${ }^{O R C 4}$ dataset (out of the 10,376 features queried in the arrays), the median number that showed an expression change in lola ${ }^{\mathrm{ORC} 4}$ was 18 (maximum 38). Thus, the set of features identified as lola-dependent in the null mutant sample provided a substantial enrichment of lola-sensitive features compared to the complete gene set, as assayed in an independent microarray experiment. This strongly supports the validity of the positives called in the original microarray analysis of the null.

Therefore, the combination of qRT-PCR of selected hits and a global validation by an independent array experiment provides strong evidence for the reliability of the identification of lola-sensitive transcripts by the microarray analysis.

\section{Gene Ontology analysis of transcripts altered in a lola null mutant}

The complete list of expression effects of lola revealed 597 features with altered labeling out of the 10, 376 features that were assayed in the experiment. Of these, 352 were increased in level in the lola mutant, implying direct or indirect negative regulation, while 245 were decreased. Consistent with the broad expression of lola itself, these features represent genes expressed in a wide

Table 1 Quantitative RT-PCR validation of array results

\begin{tabular}{|c|c|c|c|c|}
\hline Gene & Clone ID & Array fold-change (log2) & qRT-PCR fold-change (log2) & qRT-PCR $P$-value \\
\hline \multirow[t]{2}{*}{ spire } & GH13327 & 1.24 & 1.54 & $2.5 \mathrm{E}-5$ \\
\hline & SD10157 & 0.97 & & \\
\hline neural lazarillo & GH12581 & -0.56 & -2.40 & $9.3 \mathrm{E}-5$ \\
\hline genderblind/CG6070 & GH08870 & 0.29 & 0.27 & 0.016 \\
\hline \multirow[t]{3}{*}{ charybde } & LD32080 & 0.57 & 0.33 & 0.036 \\
\hline & GH09771 & 0.59 & & \\
\hline & LD22381 & 0.67 & & \\
\hline \multirow[t]{2}{*}{ walrus } & GH09945 & 0.46 & 0.27 & 0.039 \\
\hline & LD07532 & 0.44 & & \\
\hline centaurin gamma la & GM01069 & -0.95 & -0.13 & 0.25 \\
\hline blistery & SD01953 & -0.57 & 0.22 & 0.34 \\
\hline \multirow[t]{2}{*}{ bekka } & RE14959 & -0.47 & -0.46 & 0.61 \\
\hline & RE14259 & -0.52 & & \\
\hline Kr target @ 95D & SD03976 & -0.76 & 0.03 & 0.79 \\
\hline Target of rapamycin & SD02269 & -0.33 & 0.33 & 0.95 \\
\hline
\end{tabular}

Total RNA was collected from 10 to 12 hour wild type or lola mutant embryos, just as for microarray analysis, and analyzed by Taqman RT-PCR to quantify expression of ten genes that showed altered transcript level in expression arrays. A positive fold change in the table corresponds to increased accumulation of that RNA in the mutant. 


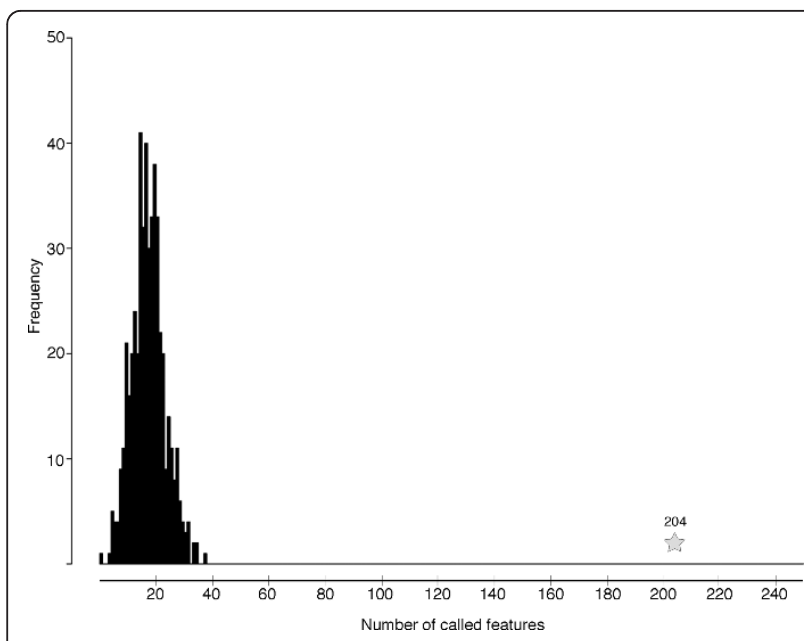

Figure 2 Validation of lola null data with an independent microarray experiment. Graphical representation of an experiment assessing the validity of the lola null mutant results by comparison to a dataset derived from an independent microarray experiment. Expression microarrays were performed comparing RNA from embryos bearing the lola single-isoform mutation lola ${ }^{\mathrm{ORC}}$ to wildtype controls [31]. The 597 features that were altered in accumulation in lola null mutant embryos were assessed for altered expression in lola ORC4: 204 of the 597 were also found to be altered in the single-isoform mutant (false discovery rate $=0.01$; grey star). In contrast, in 500 trials of a simulation in which 597 features were selected at random from the lola ${ }^{\text {ORC4 }}$ data set $(10,376$ features total) and analyzed for change in mutant versus wild type, the median number of features with altered expression in the single-isoform mutant was 18/597 (maximum 38; presented as a histogram of number of loci with altered expression versus number of occurrences of that result).

variety of tissues and at all stages of the lifecycle. Gene Ontology analysis [37], however, identifies a large number of previously characterized genes that are associated with known lola-dependent processes, including cell and axon migration and orientation, eye development, oogenesis and programmed cell death (Table 2). This analysis also reveals potential effects of lola on a variety of developmental and physiological processes for which such a role had not previously been suspected, including spermatogenesis, epithelial polarity, hormonal regulation of development, oxidative stress and aging/lifespan (Table 3).

\section{Overexpression of the actin nucleation factor Spire is an} important contributor to the lola ISNb axon stalling phenotype

Aside from retrotransposon transcripts, one of the most robust positives in the array analysis was spire. spire encodes a protein that nucleates assembly of actin filaments [38], and extensive studies of its role in
Table 2 Selected set of lola-dependent genes from microarray analysis

Axon patterning
frazzled
DSCAM
neural lazarillo
Fasciclin III
midline fasciclin
prospero
capricious
PVF-1
CBP (sarcoplasmic Ca binding prot)
sugarless
dally-like

Cell and tissue polarity

par-1

par-6

scribble

wnt-4

Signaling proteins

Cask (CaM kinase)

moesin

rhoGAP 5 A

Target of Rapamycin

rac2

Microtubules and motors
katanin80
stathmin
KLP-59C
NudC

Eye development

sickle

charlatan

asense $^{a}$

scabrous $^{a}$

rap1

roughex

Dap $160^{a}$

Lobe

twin of eyeless

fat facets

numb-associated kinase ${ }^{a}$

O-Fut- ${ }^{a}$

Cell death

grim

scylla

charybde

bunched $^{a}$

Nedd2-like caspase

Analysis of the list of loci with altered expression in a lola mutant reveals a substantial number of characterized genes with known or plausible function in established lola-dependent processes. A selection of such loci is presented here. ${ }^{a}$ Genes known to be associated with Notch signaling. 
Table 3 Enriched Gene Ontology categories from functional annotation clustering

\begin{tabular}{lc}
\hline Functional annotation & Enrichment factor \\
\hline PDZ domain-containing proteins & 1.7 \\
Aging/lifespan & 1.65 \\
Cell migration/motility & 1.65 \\
Nucleoside/nucleotide metabolism & 1.55 \\
Reactive oxygen response; oxidative stress & 1.27 \\
BESS/MADF chromatin modifying factors & 1.24 \\
Programmed cell death & 1.24 \\
Amino acid metabolism & 1.22 \\
LRR-containing proteins & 1.2 \\
GST activity & 1.18 \\
Cell-cell junctions/apicobasal polarity & 1.13 \\
Malate metabolism & 1.01 \\
Other notable annotation clusters & \\
Embryonic morphogenesis & 0.82 \\
Chemosensory behavior/olfactory learning & 0.8 \\
Hemopoiesis/hemocyte migration & 0.78 \\
Synaptogenesis/synapse organization & 0.77 \\
Post-embryonic development/imaginal disc & 0.7 \\
Gametogenesis & 0.54 \\
Spermatogenesis & 0.3 \\
Oogenesis & 0.26 \\
Axono domain-containing & 0.4 \\
RNPs & 0.28 \\
\hline
\end{tabular}

Gene Ontology analysis with DAVID [37] was used to identify processes and properties likely to be associated with lola function, based on enrichment in the collection of lola-sensitive genes. Putatively enriched annotation terms and their enrichment factors are shown, derived by functional annotation clustering performed at high stringency setting. Clusters with enrichment factors $\geq 1.3$ are supported at $P<0.05$; lower enrichment scores have proportionately lower statistical support but nonetheless are enriched in the dataset relative to background. The background set for calculating enrichment factors consisted of that subset of features on the microarray that passed the pre-processing steps and entered our statistical analysis (10, 376 features), and that could be assigned unambiguously to a single Flybase gene ID (9772 Flybase-defined genes). Retrotransposons were excluded from this analysis.

oogenesis have shown that it plays a central role in controlling cytoskeletal organization in the developing oocyte $[39,40]$. It is clear that spire also has essential roles during development since most homozygous spire mutants do not survive to adulthood, but these developmental functions of spire have never been reported. Moreover a mouse ortholog of spire is expressed in the developing CNS [41]. Given the action of spire in a different lola-regulated process and our interest in the regulation of neuronal actin organization by lola, we elected to test further the relationship of lola to spire.

We first used in situ hybridization and found that spire is expressed broadly in the embryonic central and peripheral nervous system of the fly (Figure 3 ). In the CNS, expression included the RP, aCC and VUM motoneurons, which are easy to identify individually based on their position and morphology, as well as many other cells. In the peripheral nervous system (PNS), expression was detected in all four clusters of abdominal sense organs, with highest expression in the neurons and cap cells of the chordotonal organs, and lowest expression in the v' cluster.

We next examined neural development in spire zygotic mutants and found that while the CNS axon scaffold appeared grossly normal, axons of the ISNb motonerve often failed to fully innervate the ventrolateral musculature (Figure 4). Thus, in stage 17 spir $^{1}$ mutant embryos we detected 1.64 \pm 0.09 (mean \pm standard error of the mean) neuromuscular synapses to ventral longitudinal muscles per hemisegment versus $2.73 \pm 0.03$ in wildtype $(P=3 \mathrm{E}-4)$, while in spir $^{2 F}$ we detected $1.74 \pm 0.03$ synapses $(P=2.9 \mathrm{E}-5)$.

Since both spire and lola mutants displayed defects in ISNb, we used this phenotype to test for genetic interaction between these loci. In particular, since the array and qRT-PCR analyses showed lola to be a negative regulator of spire, we wondered whether reduction of spire gene dosage would suppress the lola mutant phenotype. We again quantified the stalling of ISNb by counting the number of neuromuscular synapses formed on ventral longitudinal muscles in early stage 17 embryos.

Heterozygosity for either of two independent spire alleles significantly suppressed the lola ISNb phenotype (Figure 4E). In a heteroallelic combination of two strong lola mutations (lola ${ }^{\mathrm{ORC46/ORE76}}$ ) we detected an average of $0.30 \pm 0.06$ neuromuscular synapses per hemisegment, compared to 2.73 in wild-type embryos of this stage. Introduction of $\operatorname{spir}^{1} /^{+}$increased that to $1.20 \pm$ 0.15 synapses ( $37 \%$ suppression of the phenotype; $P=$ $0.005)$, while spir $^{2 F} /^{+}$increased it to $1.27 \pm 0.12$ synapses (40\% suppression; $P=0.002$ ). This supports the hypothesis that the approximately two-fold overexpression of spire we observe in lola mutants contributes significantly to its motoneuron stalling phenotype. The lola spir $^{-/+}$embryos sometimes looked a bit disorganized compared to other genotypes, but the significance of this is unclear.

\section{Discussion}

The transcription factor Lola is required for a variety of axon growth and guidance events in the developing fly embryo [42,43]. Expression microarray analysis of lola mutant embryos now reveals that, rather than producing large changes in the levels of a restricted number of major-effect downstream targets, Lola appears to exert its influence via the cumulative effects of small, quantitative changes in a broad spectrum of downstream loci. One key Lola target is spire, which encodes an actin nucleation factor [38] that has been studied intensively 

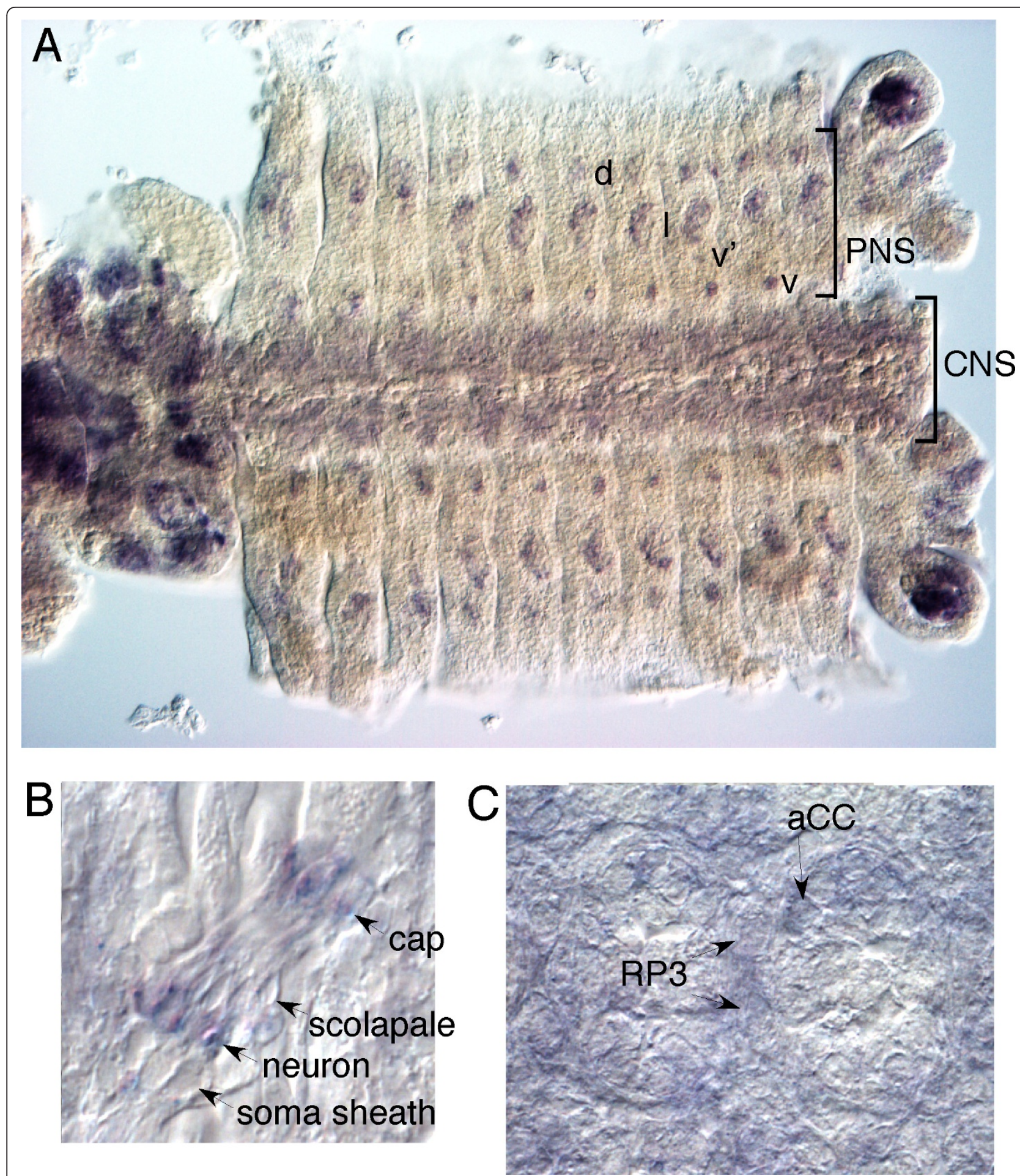

Figure 3 spire expression in the central nervous system and peripheral nervous system of Drosophila embryos. Wild-type embryos were collected, fixed, and analyzed by in situ hybridization with a probe that recognizes all spire isoforms, using alkaline phosphatase detection. Widespread expression of spire was observed in both the peripheral nervous system (PNS) and CNS of wild-type embryos. We did not discern any difference in the pattern of hybridization in lola mutant embryos. We also did not detect any difference in the strength of the spire signal, though this is not surprising given the small magnitude of the effect. (A) Filet preparation of entire embryo (stage 14). PNS and CNS are indicated with brackets. Dorsal, lateral, ventral' and ventral clusters of peripheral sensory organs are indicated as $d, l, v^{\prime}$ and $v$, respectively. Anterior is to the left. (B) High magnification view of a lateral sense organ cluster (stage 16) showing preferential expression in the neurons and cap cells of the chordotonal organs. Soma sheath and scolopale cells did not label detectably. (C) spire RNA was detected in many cells of the CNS. The motoneurons RP3 and aCC are indicated (arrows). Two segments are shown. 


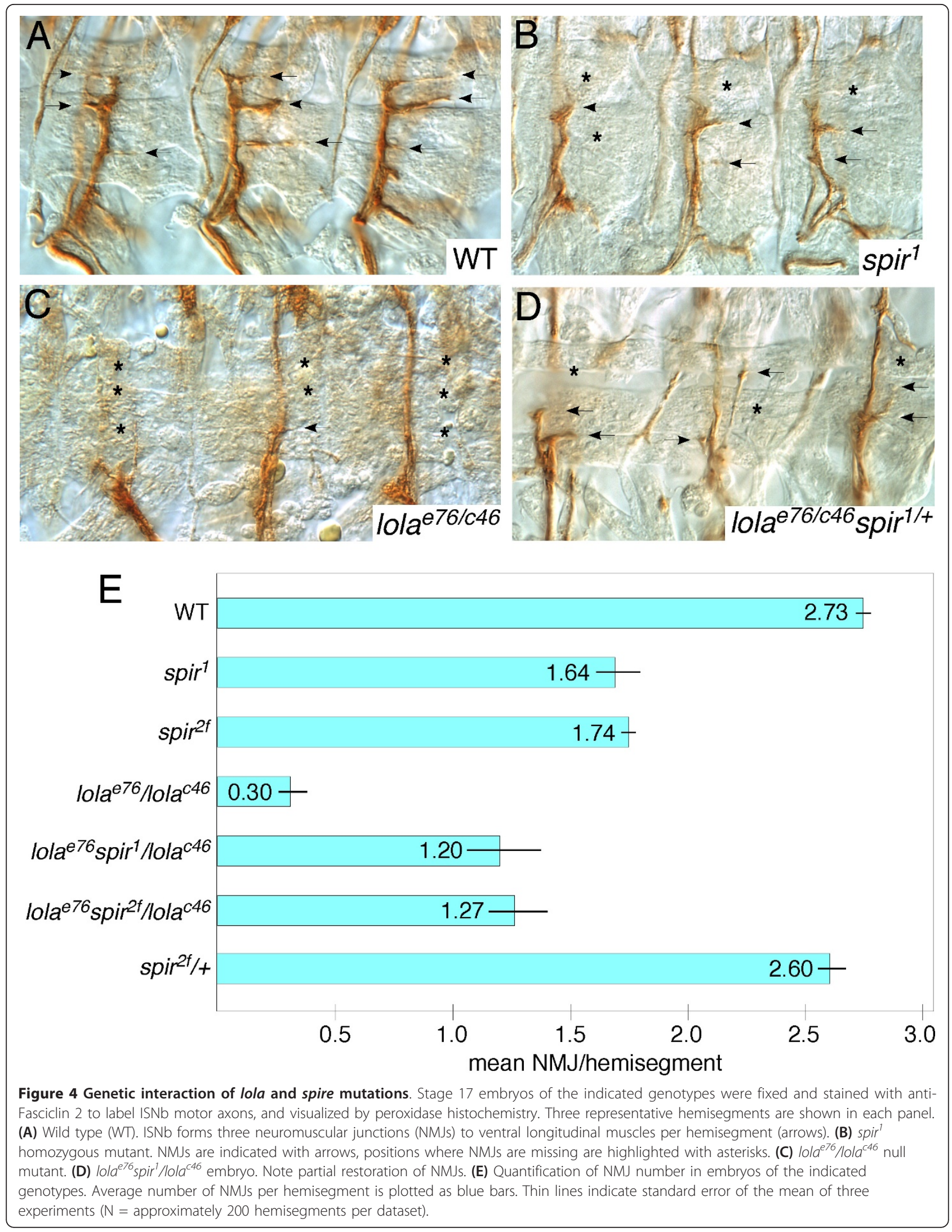


for its role in regulating cytoskeletal structure in the developing fly oocyte $[39,40]$. spire, like lola, is required for development of ISNb motor axons, its level goes up in lola mutants, and reduction of spire dosage suppresses, but does not eliminate, the ISNb mutant phenotype of lola.

Previous analysis of candidate genes implicated in various lola-dependent axon guidance processes identified several whose expression was subtly affected by lola, but none that were dramatically altered. This and other observations led to the proposition that Lola might execute its effects by fine-tuning the expression levels of genes that contribute quantitatively to various guidance decisions, and not simply by turning these genes $\mathrm{ON}$ versus OFF [17]. As an unbiased test of this hypothesis, we used expression microarrays to perform a genomewide comparison of the embryonic transcriptome of wild-type and lola zygotic mutant embryos. We analyzed RNA isolated from animals 10 to 12 hours after egg laying, at a time when a large number of lola-dependent axons are extending. By this analysis, the expression of no single-copy Drosophila gene was altered more than four-fold by lola, and few were altered more than 2.5fold. It is possible that this is an underestimate due to the compression of expression ratios in microarray experiments, but qRT-PCR results were largely consistent with the array data. It is also possible that expression of some genes may have been altered by a greater factor in just a small subset of expressing cells, but we note that most lola isoforms are themselves expressed very broadly, making this possibility less likely [26]. Finally, we know that some genes can be affected oppositely by different lola isoforms, or in different tissues [29], so it may be that a small net change in expression of a lola target gene hides larger but counteracting changes in different cells. Nonetheless, it remains that a genome-wide analysis failed to identify any single majoreffect lola target that would account for the lola axonal phenotypes. It is also true that there is a substantial maternal contribution of Lola to the embryo [42], and this may limit the measured effect of the mutation on downstream targets. We note, however, that it is the zygotic mutant phenotype of lola that we are seeking to explain, and it is therefore the quantitative effect of that zygotic mutant that is the relevant measurement for investigating the phenotype.

Microarray analysis has been widely used to identify genes associated with, or responsible for, many developmental and physiological processes. Typical analyses of expression microarray data emphasize genes whose level is strongly altered by the biological manipulation, often setting numerical cutoffs for change in expression level, together with statistical criteria, to identify true positives. In our experiments, we were compelled to eschew the use of a quantitative cutoff in fold change; for example, a commonly used criterion of a two-fold minimum change would have excluded from analysis all but 26 single-copy genes in the genome. Rather, the nature of the biological process we studied, and the nature of lola, required that we minimize the biological and technical variance to achieve exceptionally tight statistics. In the end, qRT-PCR validation of expression changes from 1.2-fold (genderblind) to 2.5-fold (spire) provided support for $50 \%$ of the putative downstream effects of lola. We note that this is likely to be an underestimate of the reliability of the array results since at these small folddifferences we were at or beyond the usual sensitivity of RT-PCR itself, and it is as likely that RT-PCR was reporting false negatives as the microarrays were reporting false positives. Validity of the results was also supported more globally by independent expression profiling of another lola allele. Thus, these data underscore the efficacy of microarray analysis for detecting reliably even quite small changes in expression level.

Known genes whose expression was altered in the lola mutant shed light on many lola-dependent processes. Previous experiments had led to the notion that lola likely co-regulates a suite of interacting genes that are important for particular axon guidance decisions, and indeed, we find expression of a number of well-characterized guidance receptors to be altered in lola. frazzled, which was identified as a downstream target of lola, is on its own known to be required for three lola-dependent axonal processes: ISNb development in the periphery, and both longitudinal and commissural axon extension in the CNS [44]. Among other factors downstream of lola are midline fasciclin (longitudinal and commissural axons) [45], fasciclin 3 and capricious (ISNb) [46,47] and neural lazarillo (thought to be involved in both longitudinal and commissural axon guidance) [48]. Also identified were genes for a number of ligands, receptors and receptor-modifying proteins not previously associated with lola-dependent processes, such as sugarless, dallylike, wnt $4 a$ and PVF-1. It now becomes interesting to investigate the potential role of these genes in axon patterning, and in migration and orientation of sensory neurons. Aside from cell surface and extracellular proteins, expression of genes encoding a number of intracellular signaling proteins was found to be altered, including prospero (which in hypomorphic alleles produces a phenotype very similar to that of lola (EG, unpublished observations)), as well as moesin, Rac2, and a calmodulin-dependent protein kinase $(C A K I)$. An unexpected cluster of downstream effects comprised genes for proteins modulating microtubule structure and function, including katanin, stathmin, NudC and KLP-59C. lola also interacts genetically with the axon patterning function and other activities of the 
receptor Notch [49,50] (EG, unpublished observations), and we find a cluster of affected genes that modulate Notch action, including sca, Nak, Dap-160 and O-fut1. In addition to these known genes, Gene Ontology analysis [37] identifies a large number of lola-dependent loci that have not yet been characterized in the fly, but whose annotations cluster with lola-dependent genes of known function. This provides a substantial list of excellent candidates for additional contributors to loladependent processes. Unfortunately, the large number of Lola isoforms, and their heteromeric combinations, makes it impossible for us to extract Lola binding site consensus sequences from these candidates using standard computational approaches. Extensive molecular experiments will be necessary in the future to identify response elements for individual heteromeric forms of Lola.

lola has several characterized functions outside of axon patterning. For example, it affects cell fates in the eye [50], and indeed, there is a substantial group of eye patterning genes included in the list of lola-affected loci (sickle, charlatan, asense, rap, roughex, Lobe, target of eyeless and fat facets). Additionally, consistent with the role of lola in controlling programmed cell death during oogenesis [25], we find grim, scylla, charybde, bunched and Nedd2-like caspase among the downstream effects. It should be noted that since our microarray analysis was performed only with mid-stage embryos, we cannot distinguish whether the effects of lola on these postembryonic processes are mediated by the same downstream targets that we see affected during embryogenesis. Seeing that these genes can be modulated by lola at one stage of the lifecycle, however, makes them more attractive candidates for analysis at other stages. Finally, in addition to genes affecting known lola-dependent processes, the set of genes altered in lola mutants identifies clusters associated with new processes that would be worth investigating for a role of lola. These include aging, oxidative stress, hormonal regulation of development, tracheal development and maintenance, cell polarity and olfactory learning, among others.

One of the most robust putative downstream effects we identified for lola was downregulation of the actin nucleation factor Spire. This was immediately striking since spire is known to be a critical regulator of the oocyte cytoskeleton during Drosophila oogenesis. spire is required for both anteroposterior and dorsoventral patterning of the developing oocyte [30]. By modulating actin structure, Spire restrains bundling of oocyte microtubules, thereby blocking cytoplasmic streaming in the oocyte until critical antero-posterior and dorso-ventral polarity cues become stably bound to cortical anchoring sites or initiate irreversible signaling cascades $[39,40]$. At the biochemical level, Spire nucleates actin filaments by bringing together actin monomers to assemble a filament nucleus [38], and it may then transfer this nucleus to the associated formin, Cappuccino, which stimulates filament growth [51]. While the developmental function of spire has been studied most thoroughly in the oocyte, strong mutations in this gene are largely lethal, with only small numbers of escapers surviving to adulthood, and this suggested the existence of as yet uncharacterized zygotic functions of spire. Moreover, a mouse ortholog of spire is expressed in the developing and adult brain [41]. Here we found that spire is required for a well-characterized lola-dependent neuronal process, extension of the ISNb motonerve. ISNb was an ideal candidate for the sort of function we had previously hypothesized for lola, since it is known to depend on the summed, quantitative effects of a large collection of regulators. We therefore exploited ISNb to examine more carefully the potential interaction of lola and spire, and found that genetic reduction of spire suppressed the ISNb mutant phenotype of lola, consistent with the upregulation of spire in a lola mutant making a significant contribution to ISNb axon stalling in lola. By itself, expression analysis cannot distinguish whether spire is a direct target of Lola or whether the upregulation of spire message is a downstream consequence of other changes set in motion by lola. Further biochemical studies of the DNA binding properties of Lola isoforms will be necessary to assess this. Finally, lola has other phenotypes that are not suppressed by reduction of spire. These may reflect, for example, roles of loladependent guidance molecules that are themselves spire -independent, or the action of Spire-independent aspects of growth cone signaling.

Efforts to mimic the lola ISNb phenotype by overexpression of spire were not successful. There are several possible reasons for this. First, there are thought to be at least eight Spire protein isoforms, based on cDNA and expressed sequence tag data (Flybase), and it may be that a particular combination of isoforms, or a specific ratio of expression levels of different isoforms, is necessary to give the ISNb stalling phenotype. Alternatively, it may be that this phenotype is produced only when spire upregulation occurs in the context of some other downstream effect(s) of lola. Additional experiments will be necessary to discriminate among these models.

Superficially, it seems remarkable that complete loss of spire causes stalling of ISNb axons, yet the upregulation of spire that occurs in a lola mutant also contributes to ISNb stalling. Evidently, excessive nucleation of actin filaments from spire overexpression is as detrimental to growth cone motility as is the failure of actin nucleation from absence of the protein. We and others have observed similar non-linearity in the effects of a number 
of signaling and cytoskeletal regulatory proteins in other axon guidance paradigms, and it appears to be a common feature of the relationship of signaling to morphogenesis. Thus, for example, even though Abl tyrosine kinase pathway signaling appears to be essential for most axon growth [52], extension of longitudinal pioneer axons of the fly CNS requires suppression of Abl signaling to achieve the proper balance in the steps of actin dynamics [53]. Similarly, for the Rac GTPases, expression of dominant negative and dominant constitutive forms of the protein are equally effective for inhibiting axon motility, but in one case from excessive stabilization of actin filaments and in the other from insufficient stabilization [54]. Spire now provides another example of this generalization, and underscores the need for signaling networks to evoke a balance in the steps in actin dynamics, thus optimizing throughput through the mechanical cycle of growth cone motility [55].

lola mutants have profound effects on axon patterning, even though systematic molecular analysis reveals only subtle modulation of downstream target gene expression. This observation highlights the exquisite sensitivity of motility and guidance to the balance among cell signaling networks, and thus also to the gene expression mechanisms that set the boundaries of that balance.

\section{Conclusions}

We have used a genome-wide analysis to identify the suite of genes whose expression is altered in embryos lacking the Drosophila transcription factor Lola. Gene Ontology analysis sheds light on the regulation of several characterized lola functions, including axon guidance, synapse formation, eye development and oogenesis, by revealing the lola-dependence of genes known to be involved in those processes, and also by identifying a large number of previously uncharacterized lola-dependent genes that are likely to contribute to these processes. Additionally, these results identify novel processes that are likely to be regulated by lola. Regarding axon patterning, our analysis reveals that Lola suppresses expression of the actin nucleation factor Spire, and this is crucial for its ability to promote growth of motor axons in vivo. These data underscore the critical importance of ensuring the correct levels of actin regulatory proteins in a cell to promote motility effectively.

\section{Materials and methods}

\section{Genetics}

Fly genetics and husbandry followed standard methods. spire mutant alleles were obtained from the Bloomington Drosophila Stock Center. For RNA extraction, mutant embryos were $w^{1118}$; lola ${ }^{\text {ORE76 }}$ sca-GAL4/lola ${ }^{\text {ORE76 }} U A S-\tau$ eGFP; controls were $w^{1118}$; sca-GAL4/UAS- $\tau$-eGFP.
Numbering of lola isoforms follows established nomenclature $[26,27]$. We note that since the same lola allele was used to generate both the sca-GAL4 and UAS- $\tau-G F P$ recombinant chromosomes, we cannot exclude the possibility that some of the gene expression effects we observed are due to modifier loci on the lola chromosome.

\section{Embryo collection and RNA preparation}

Embryos were collected for 2 hours at $25^{\circ} \mathrm{C}$ on grape juice agar plates and incubated an additional 6 hours at $25^{\circ} \mathrm{C}$ in a moist chamber. Embryos of the desired genotype were then hand-sorted with a fluorescent dissecting microscope based on positive GFP expression in the CNS. Sorted embryos were returned to $25^{\circ} \mathrm{C}$ and allowed to develop until 10.0 hours after the end of egg collection. lola mutant and control embryos were collected concurrently. Seven independent sample pairs were used for microarray experiments and three to five for qRT-PCR.

Collected embryos were dechorionated with 50\% bleach, rinsed once with $0.1 \mathrm{M} \mathrm{Na}$ phosphate $\mathrm{pH} 7.2$, $0.3 \%$ Triton $\mathrm{X}-100$, and rinsed twice with sterile water. Total RNA was then extracted with Trizol (Invitrogen, Carlsbad, CA, USA) per the manufacturer's instructions. The final RNA pellet was rinsed twice with $75 \%$ ethanol, air-dried for 10 minutes, resuspended in $20 \mu \mathrm{l}$ RNasefree water at $60^{\circ} \mathrm{C}$ and stored at $-70^{\circ} \mathrm{C}$. Each RNA sample for microarrays comprised 20 to $30 \mu \mathrm{g}$ of total RNA, derived from approximately 300 embryos; samples for RT-PCR were derived from 50 embryos.

\section{Array methods}

Preparation of spotted cDNA arrays bearing the Drosophila Gene Collection (release 1 and release 2), RNAlabeling, hybridization, scanning and extraction of feature intensities using GenePix were performed by the Fred Hutchinson Cancer Research Center (FHCRC) Genome Analysis Facility as described elsewhere [32]. Of the seven RNA pairs that were analyzed for each array experiment, in four cases the mutant sample was labeled with $\mathrm{Cy} 3$ and control with $\mathrm{Cy} 5$; in the remaining three cases the labels were reversed. Microarray data have been deposited to the NCBI GEO database.

\section{Statistical methods for analysis of array data}

Array data analysis was performed with limma [34,35] in the $\mathrm{R}$ statistical programming language ( $\mathrm{R}$ Development Core Team) [56]. Briefly, spot intensity data (from GenePix) was read-in using the limma function read. maimages. No background correction was applied, within-array intensity values were normalized using print tip loess, and between-array intensity values were normalized using scale. Gene-wise linear models to the normalized intensity ratios were fitted using limFit with 
default parameters. Several statistics, including the moderated $\mathrm{t}$-statistic and the log-odds of differential expression, were calculated for each array probe using eBayes. The moderated $t$-statistics were classified as significant using an adjusted $P$-value of 0.05 . Adjustment for multiple testing was done using the Benjamini-Hochberg method for controlling the false discovery rate (limma function decideTests, with adjust.method $=$ ' $\mathrm{BH}$ '). Spots were excluded from analysis if no corresponding sequence was available from public databases, if FHCRC production notes indicated spot contamination or if GenePix data extraction software failed to locate the probe in any experiment. In total, 10, 376 features were included in the analysis.

\section{Quantitative real time PCR}

Real time PCR was performed on an Applied Biosystems 7300 Real-Time PCR System using Taqman Gene Expression Assays (Applied Biosystems, Foster City, CA, USA). Primer sets were purchased commercially (Applied Biosystems) as follows: Ribosomal protein L32 (RPL32; Dm02151827_g1); Bekka (Dm02363268_s1); blistery (Dm02138346_m1); CG6070 (Dm02145281_m1); charybde (Dm01802349_m1); Kruppel target at 95D (Dm02150605_m1); Neural Lazarillo (Dm01844577_g1); spire (Dm01811138_m1); Target of rapamycin (Dm01843300_g1); and walrus (Dm01792969_g1). Gene expression levels were normalized to the endogenous control RpL32 using the standard curve method according to the manufacturer's instructions. Normalized gene expression levels of lola null mutant samples were measured relative to wild-type control. Measurements were repeated with three to five biological replicates, and each biological replicate was performed with three technical replicates.

\section{Histochemistry and microscopy}

Embryos for in situ hybridization and protein immunostaining were prepared and examined by standard methods [26]. Anti-Fasciclin 2 was from the University of Iowa Developmental Studies Hybridoma Bank, the biotin anti-mouse secondary was from Jackson ImmunoResearch (West Grove, PA, USA) and detection was with Vectastain (Vector Labs, Burlingame, CA, USA \{ Reagents for in situ hybridization and alkaline phosphatase detection were from Roche Applied Sciences (Indianapolis, IN, USA).

ISNb phenotype was quantified by labeling stage 17 embryos with anti-Fasciclin 2, using DAB detection, and counting the number of neuromuscular junctions to ventral longitudinal muscles (muscles 7, 6, 13 and 12) in segments A2 to A7 in fillet preparations. Embryo genotypes were determined unambiguously using gratuitous markers. Statistical significance was assessed by t-test; N = approximately 200 hemisegments per dataset.

\section{Additional material}

\begin{abstract}
Additional file 1: Table S1. Complete list of array features reporting altered RNA level in Iola ${ }^{\text {ORE76 }}$ mutant embryos. The table lists all probes from the Drosophila Gene Collection that reported significantly altered RNA accumulation in lola null mutant embryos versus the matched wild-type control (ordered by $P$-value). BLAST analysis was used to assign each probe to the corresponding Drosophila gene where possible; these are given by FBgn number and locus name. Fold change is reported as $\log 2$ and statistical support is reported as adjusted $P$-value [35]. Note that lola ORE76 is a nonsense mutation, and indeed, features corresponding to lola isoforms reported only modest changes in transcript level. Unfortunately, expression of slit, robo and robo3 could not be assayed in this experiment as they were either absent from this release of the Drosophila Gene Collection or found to be defective during chip preparation. robo2 did not show a significant change in RNA accumulation.
\end{abstract}

\section{Abbreviations}

CNS: central nervous system; FHCRC: Fred Hutchinson Cancer Research Center; GFP: green fluorescent protein; PNS: peripheral nervous system; qRTPCR: quantitative RT-PCR.

\section{Acknowledgements}

We thank all the members of our lab for their advice and assistance with these experiments. For much helpful advice we also thank Celeste Berg, Cecilia Moens, Tom Reh, Kathleen Kerr, Ben Feldman, Chi-Hon Lee and Ward Odenwald, and for comments on the manuscript we thank Katie Kerr, Don van Meyel, Akinao Nose and Brian Oliver. We thank the Berkeley Drosophila Genome Project for the gift of the Drosophila Gene Collection. We are particularly grateful to Jeff Delrow and the Microarray Facility of the FHCRC for their assistance in designing, performing and analyzing the microarray experiments. These experiments were supported in part by NSF grant IBN9904519 to EG, and by funds from the Basic Neuroscience Program of the Intramural Research Program NINDS, NIH (Z01 NS003013).

\section{Author details}

${ }^{1}$ Basic Neuroscience Program, National Institutes of Neurological Disorders and Stroke, National Institutes of Health, Bethesda, MD 20892, USA. ${ }^{2}$ National Human Genome Research Institute, National Institutes of Health, Bethesda, MD, 20892 USA. ${ }^{3}$ Fred Hutchinson Cancer Research Center, Seattle, WA, 98109 USA. ${ }^{4}$ Graduate Program in Molecular and Cellular Biology, University of Washington and Fred Hutchinson Cancer Research Center, Seattle, WA, 98109 USA.

\section{Authors' contributions}

MAG performed the microarray analysis and validation, RK performed the genetic validation and characterization of spire expression, EG was responsible for overall design and contributed to interpretation of the results. All three authors contributed to writing of the manuscript and all read and approved its final form.

\section{Competing interests}

The authors declare that they have no competing interests.

Received: 7 September 2011 Accepted: 30 November 2011 Published: 30 November 2011

\section{References}

1. Butler SJ, Tear G: Getting axons onto the right path: the role of transcription factors in axon guidance. Development 2007, 134:439-448.

2. Polleux F, Ince-Dunn G, Ghosh A: Transcriptional regulation of vertebrate axon guidance and synapse formation. Nat Rev Neurosci 2007, 8:331-340. 
3. Simon M: Receptor tyrosine kinases: Specific outcomes from general signals. Cell 2000, 103:13-15.

4. Tanabe $Y$, Jessell TM: Diversity and pattern in the developing spinal cord. Science 1996, 274:1115-1123.

5. Ghysen A, Jan LY, Jan YN: Segmental determination in Drosophila central nervous system. Cell 1985, 40:943-948.

6. Gould AP, White RA: Connectin, a target of homeotic gene control in Drosophila. Development 1992, 116:1163-1174.

7. Thor S, Andersson SG, Tomlinson A, Thomas JB: A LIM-homeodomain combinatorial code for motor-neuron pathway selection. Nature 1999, 397:76-80.

8. Thor S, Thomas JB: The Drosophila islet gene governs axon pathfinding and neurotransmitter identity. Neuron 1997, 18:397-409.

9. Tsuchida T, Ensini M, Morton SB, Baldassare M, Edlund T, Jessell TM, Pfaff SL: Topographic organization of embryonic motor neurons defined by expression of LIM homeobox genes. Cell 1994, 79:957-970.

10. Labrador JP, O'Keefe D, Yoshikawa S, McKinnon RD, Thomas JB, Bashaw GJ: The homeobox transcription factor even-skipped regulates netrinreceptor expression to control dorsal motor-axon projections in Drosophila. Curr Biol 2005, 15:1413-1419.

11. Yang L, Garbe DS, Bashaw GJ: A frazzled/DCC-dependent transcriptional switch regulates midline axon guidance. Science 2009, 324:944-947.

12. Winberg ML, Mitchell KJ, Goodman CS: Genetic analysis of the mechanisms controlling target selection: complementary and combinatorial functions of netrins, semaphorins, and IgCAMs. Cell 1998, 93:581-591.

13. Kaufmann N, Wills ZP, Van Vactor D: Drosophila Rac1 controls motor axon guidance. Development 1998, 125:453-461.

14. Wills Z, Bateman J, Korey CA, Comer A, Van Vactor D: The tyrosine kinase $\mathrm{Abl}$ and its substrate enabled collaborate with the receptor phosphatase Dlar to control motor axon guidance. Neuron 1999, 22:301-312.

15. Wills Z, Marr L, Zinn K, Goodman CS, Van Vactor D: Profilin and the Abl tyrosine kinase are required for motor axon outgrowth in the Drosophila embryo. Neuron 1999, 22:291-299.

16. Garbe DS, Bashaw GJ: Axon guidance at the midline: from mutants to mechanisms. Crit Rev Biochem Mol Biol 2004, 39:319-341.

17. Crowner D, Madden K, Goeke S, Giniger E: Lola regulates midline crossing of CNS axons in Drosophila. Development 2002, 129:1317-1325.

18. Madden K, Crowner D, Giniger E: Iola has the properties of a master regulator of axon-target interactions for SNb motor axons of Drosophila. Dev Biol 1999, 213:301-313.

19. Brenman JE, Gao FB, Jan $L Y$, Jan $Y N$ : Sequoia, a tramtrack-related zinc finger protein, functions as a pan-neural regulator for dendrite and axon morphogenesis in Drosophila. Dev Cell 2001, 1:667-677.

20. Spletter ML, Liu J, Su H, Giniger E, Komiyama T, Quake S, Luo L: Lola regulates Drosophila olfactory projection neuron identity and targeting specificity. Neural Dev 2007, 2:14.

21. Edwards AC, Zwarts L, Yamamoto A, Callaerts P, Mackay TF: Mutations in many genes affect aggressive behavior in Drosophila melanogaster. BMC Biol 2009, 7:29

22. Mackay TF, Heinsohn SL, Lyman RF, Moehring AJ, Morgan TJ, Rollmann SM: Genetics and genomics of Drosophila mating behavior. Proc Natl Acad Sci USA 2005, 102(Suppl 1):6622-6629.

23. Morozova TV, Anholt RR, Mackay TF: Transcriptional response to alcohol exposure in Drosophila melanogaster. Genome Biol 2006, 7:R95.

24. Yamamoto A, Zwarts L, Callaerts P, Norga K, Mackay TF, Anholt RR: Neurogenetic networks for startle-induced locomotion in Drosophila melanogaster. Proc Natl Acad Sci USA 2008, 105:12393-12398.

25. Bass BP, Cullen $K$, McCall $K$ : The axon guidance gene lola is required for programmed cell death in the Drosophila ovary. Dev Biol 2007, 304:771-785.

26. Goeke S, Greene EA, Grant PK, Gates MA, Crowner D, Aigaki T, Giniger E: Alternative splicing of lola generates 19 transcription factors controling axon guidance in Drosophila. Nat Neurosci 2003, 6:917-924.

27. Ohsako T, Horiuchi T, Matsuo T, Komaya S, Aigaki T: Drosophila lola encodes a family of BTB-transcription regulators with highly variable Cterminal domains containing zinc finger motifs. Gene 2003, 311:59-69.

28. Zhang W, Wang Y, Long J, Girton J, Johansen J, Johansen KM: A developmentally regulated splice variant from the complex lola locus encoding multiple different zinc finger domain proteins interacts with the chromosomal kinase JIL-1. J Biol Chem 2003, 278:11696-11704.
29. Cavarec L, Jensen S, Casella JF, Cristescu SA, Heidmann T: Molecular cloning and characterization of a transcription factor for the copia retrotransposon with homology to the BTB-containing lola neurogenic factor. Mol Cell Biol 1997, 17:482-494.

30. Manseau LJ, Schupbach T: cappuccino and spire: two unique maternaleffect loci required for both the anteroposterior and dorsoventral patterns of the Drosophila embryo. Genes Dev 1989, 3:1437-1452.

31. Gates MA: From transcription to axon guidance: Uncovering downstream effectors of longitudinals lacking in Drosophila melanogaster. PhD thesis University of Washington and Fred Hutchinson Cancer Research Center, Program in Molecular and Cell Biology; 2008.

32. Pierce SB, Yost C, Anderson SA, Flynn EM, Delrow J, Eisenman RN: Drosophila growth and development in the absence of $d M y c$ and $d M n t$. Dev Biol 2008, 315:303-316.

33. Stapleton M, Liao G, Brokstein P, Hong L, Carninci P, Shiraki T, Hayashizaki Y, Champe M, Pacleb J, Wan K, Yu C, Carlson J, George R, Celniker S, Rubin GM: The Drosophila gene collection: identification of putative fulllength cDNAs for $70 \%$ of D. melanogaster genes. Genome Res 2002, 12:1294-1300

34. Smyth GK, Speed T: Normalization of CDNA microarray data. Methods 2003, 31:265-273.

35. Smyth GK: Linear models and empirical Bayes methods for assessing differential expression in microarray experiments. Stat Appl Genet Mol Biol 2004, 3:Article 3

36. Benjamini $Y$, Hochberg Y: Controlling false discovery rate: A practical and powerful approach to multiple testing. J Royal Statistical Soc B 1995, 57:289-300.

37. Huang DW, Sherman BT, Lempicki RA: Systematic and integrative analysis of large gene lists using DAVID bioinformatics resources. Nat Protoc 2009, 4:44-57.

38. Quinlan ME, Heuser JE, Kerkhoff E, Mullins RD: Drosophila Spire is an actin nucleation factor. Nature 2005, 433:382-388

39. Rosales-Nieves AE, Johndrow JE, Keller LC, Magie CR, Pinto-Santini DM, Parkhurst SM: Coordination of microtubule and microfilament dynamics by Drosophila Rho1, Spire and Cappuccino. Nat Cell Biol 2006, 8:367-376.

40. Theurkauf WE: Premature microtubule-dependent cytoplasmic streaming in cappuccino and spire mutant oocytes. Science 1994, 265:2093-2096.

41. Schumacher N, Borawski JM, Leberfinger CB, Gessler M, Kerkhoff E: Overlapping expression pattern of the actin organizers Spir-1 and formin-2 in the developing mouse nervous system and the adult brain. Gene Expr Patterns 2004, 4:249-255.

42. Giniger $\mathrm{E}$, Tietje $\mathrm{K}$, Jan $\mathrm{LY}$, Jan $\mathrm{YN}$ : Iola encodes a putative transcription factor required for axon growth and guidance in Drosophila. Development 1994, 120:1385-1398.

43. Seeger M, Tear G, Ferres-Marco D, Goodman CS: Mutations affecting growth cone guidance in Drosophila: Genes necessary for guidance towards or away from the midline. Neuron 1993, 10:409-426.

44. Kolodziej PA, Timpe LC, Mitchell KJ, Fried SR, Goodman CS, Jan LY, Jan YN: frazzled encodes a Drosophila member of the DCC immunoglobulin subfamily and is required for CNS and motor axon guidance. Cell 1996, 87:197-204.

45. Hu S, Sonnenfeld M, Stahl S, Crews ST: Midline Fasciclin: a Drosophila Fasciclin---related membrane protein localized to the CNS midline cells and trachea. J Neurobiol 1998, 35:77-93.

46. Chiba A, Snow P, Keshishian H, Hotta Y: Fasciclin III as a synaptic target recognition molecule in Drosophila. Nature 1995, 374:166-168.

47. Shishido E, Takeichi M, Nose A: Drosophila synapse formation: regulation by transmembrane protein with Leu-rich repeats, CAPRICIOUS. Science 1998, 280:2118-2121.

48. Sanchez D, Ganfornina MD, Torres-Schumann S, Speese SD, Lora JM, Bastiani MJ: Characterization of two novel lipocalins expressed in the Drosophila embryonic nervous system. Int J Dev Biol 2000, 44:349-359.

49. Ferres-Marco D, Gutierrez-Garcia I, Vallejo DM, Bolivar J, Gutierrez-Avino FJ, Dominguez M: Epigenetic silencers and Notch collaborate to promote malignant tumours by Rb silencing. Nature 2006, 439:430-436.

50. Zheng L, Carthew RW: Lola regulates cell fate by antagonizing Notch induction in the Drosophila eye. Mech Dev 2008, 125:18-29.

51. Vizcarra CL, Kreutz B, Rodal AA, Toms AV, Lu J, Zheng W, Quinlan ME, Eck MJ: Structure and function of the interacting domains of Spire and Fmn-family formins. Proc Natl Acad Sci USA 2011, 108:11884-11889. 
52. Hoffmann FM: Drosophila $a b l$ and genetic redundancy in signal transduction. TIGS 1991, 7:351-355.

53. Kuzina I, Song JK, Giniger E: How Notch establishes longitudinal axon connections between successive segments of the Drosophila CNS. Development 2011, 138:1839-1849.

54. Luo L, Liao J, Jan LY, Jan YN: Distinct morphogenetic functions of similar small GTPases Drosophila Drac1 is involved in axonal outgrowth and myoblast fusion. Genes Dev 1994, 8:1787-1802.

55. Giniger E: How do Rho family GTPases direct axon growth and guidance? A proposal relating signaling pathways to growth cone mechanics. Differentiation 2002, 70:385-396.

56. The R Project for Statistical Computing. [http://www.R-project.org].

doi:10.1186/1749-8104-6-37

Cite this article as: Gates et al:: A genome-wide analysis reveals that the Drosophila transcription factor, Lola, promotes axon growth in part by suppressing expression of the actin nucleation factor, Spire. Neural Development 2011 6:37.

\section{Submit your next manuscript to BioMed Central} and take full advantage of:

- Convenient online submission

- Thorough peer review

- No space constraints or color figure charges

- Immediate publication on acceptance

- Inclusion in PubMed, CAS, Scopus and Google Scholar

- Research which is freely available for redistribution

Submit your manuscript at www.biomedcentral.com/submit 